\title{
Spin polarized and degenerate tunneling spectra in intrinsic Josephson junctions of Bi2212
}

\author{
M. Ozdemir ${ }^{1}$, L. Ozyuzer*1, and C. Kurter ${ }^{2}$ \\ ${ }^{1}$ Department of Physics, Izmir Institute of Technology, Izmir, 35430, Turkey \\ 2 Materials Science Division, Argonne National Laboratory, Argonne, IL 60439, USA
}

Received 30 July 2006, revised 25 September 2006, accepted 25 September 2006

Published online 7 February 2007

PACS 72.25.-b, 74.50.+r, 74.81.Fa

Tunneling characteristics of $\mathrm{HgBr}_{2}$ intercalated superconducting Bi2212 single crystals have been obtained by using $10 \times 10 \mu \mathrm{m}^{2}$ intrinsic Josephson junction stacks, so called mesa structures. The spin degenerate current is driven along the $c$-axis with Au layer using point contact tunneling assembly at $4.2 \mathrm{~K}$. The spin polarized current is also driven along the $c$-axis of crystals with $\mathrm{Au} / \mathrm{Co} / \mathrm{Au}$ multilayer. In order to understand the role of ferromagnetic layer (Co), quasiparticle branches are examined with and without magnetic field. The magnetic field evolution of switching currents are obtained for gaining further insight about the spin injection through the stack.

C 2007 WILEY-VCH Verlag GmbH \& Co. KGaA, Weinheim

Superconducting Bi2212 single crystals, which are naturally grown $\mathrm{CuO}_{2}$ superlattices separated by $\mathrm{Bi}-\mathrm{O}$ and $\mathrm{Sr}-\mathrm{O}$ layers, behave as intrinsic Josephson junctions (IJJ) along the $c$-axis [1]. This has provided unique opportunities in terms of understanding physics of high temperature superconductors (HTSs) and creating new devices from these materials. IJJ tunneling spectroscopy can be used as a complimentary tool to evaluate the results of point contact tunneling [2], break junction [3] and STM/S [4] measurements. On the other hand, IJJ tunneling suffers from overinjection of quasiparticles, and this results in heating and nonequilibrium effects [5-8]. One of the effective solutions to minimize these effects is to decrease the coupling between $\mathrm{CuO}_{2}$ layers by intercalation of $\mathrm{Bi} 2212$ with an inert molecule such as $\mathrm{HgBr}_{2}, \mathrm{I}_{2}, \mathrm{HgI}_{2}$. IJJ tunneling spectroscopy studies of $\mathrm{HgBr}_{2}$ intercalated crystals can reveal crucial features [9] obtained from conventional tunnel junctions such as dip and hump which are linked to mechanism of superconductivity [10]. Recent advances in IJJ research have focused on utilizing superconductors in spintronics (spin-based electronics) and heterostructure junctions have been fabricated for spin-dependent transport experiments. These experiments have been known to a good probe to analyze the physical properties of ferromagnetic and superconducting materials [11].

In this study, the tunneling spectra of a regular mesa-IJJ stack $(58 \mathrm{~nm})$ with a Au layer and a ferromagnetic mesa-IJJ stack $\left(73 \mathrm{~nm}\right.$ ) with $\mathrm{Au} / \mathrm{Co} / \mathrm{Au}$ multilayer on $\mathrm{HgBr}_{2}$ intercalated $\mathrm{Bi} 2212$ single crystals are reported with and without magnetic field application. Insets of Figs. 1 (a) and (b) show schematic side view of the fabricated structures. In the latter configuration, ferromagnetic metal, Co is used as a source of spin polarized carriers injected into Bi2212 crystal. The aim of this study is to investigate the effect of the spin transfer from ferromagnetic metal through the Bi2212 stack and to obtain the influence of such kind of effect on the $c$-axis tunneling characteristics. For this reason, two sets of samples were prepared; in one set, freshly cleaved Bi2212 crystals have been deposited with Au while the other set has ferromagnetic multilayer $(\mathrm{Au}=15 \mathrm{~nm} / \mathrm{Co}=80 \mathrm{~nm} / \mathrm{Au}=156 \mathrm{~nm})$ on the crystal. Using photolithography and $\mathrm{Ar}$ ion beam etching techniques described elsewhere [12], $10 \times 10 \mu \mathrm{m}^{2}$ mesa structures were fabricated on $\mathrm{HgBr}_{2}$ intercalated crystals with $\mathrm{T}_{c}=74 \mathrm{~K}$.

* Corresponding author: e-mail: ozyuzer@ iyte.edu.tr, Phone: +90 2327507518 , Fax: +90 2327507509 

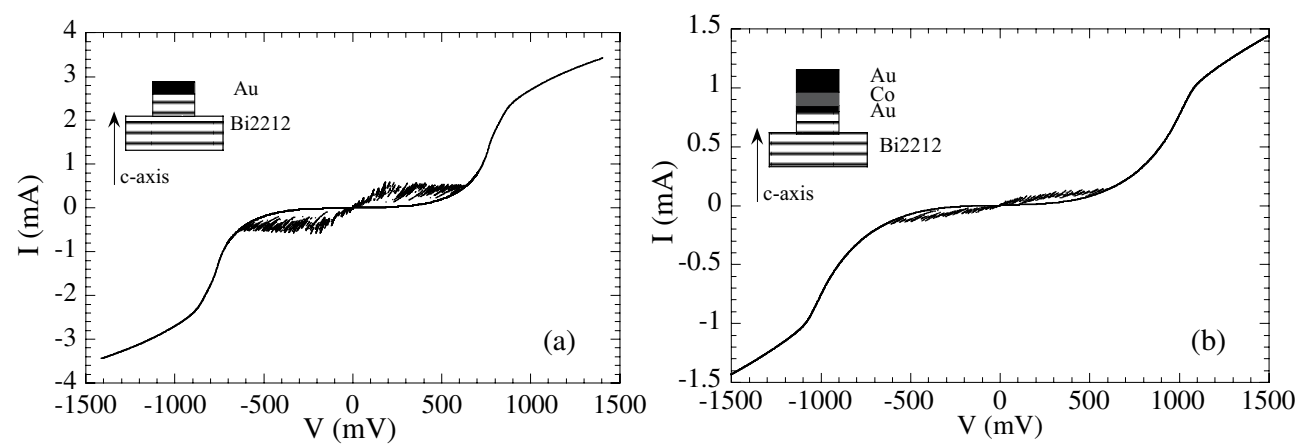

Fig. 1 The $I-V$ characteristics of $10 \times 10 \mu \mathrm{m}^{2}$ mesas with (a) merely gold layer (spin degenerate configuration) (b) ferromagnetic multilayer (spin polarized configuration). The insets show the side view of mesas.

Figure 1(a) and (b) show the $I-V$ characteristics of $10 \times 10 \mu \mathrm{m}^{2}$ mesas on intercalated crystals at 4.2 $\mathrm{K}$ for spin degenerate and spin polarized configuration respectively without any applied magnetic field. In regular mesa configuration, the quasiparticle branches do not show so much difference in switching current up to sumgap branches while ferromagnetic mesa characteristics exhibit gradual distribution in quasiparticle branches due to self-field of ferromagnetic Co layer. However, high bias branches are not affected by injected spin polarized current as much as low bias characteristics. Because the polarized quasiparticles loose their spin-direction memory as they move away from spin injector, Co layer. This may help for finding the spin diffusion length of Bi2212 when tall IJJ stacks are prepared.

Figures 2(a) and (b) exhibit magnetic field evolution of $I-V$ dependences for two configurations from 0 $\mathrm{G}$ to $1000 \mathrm{G}$ for subgap regions. Two figures differ from each other in that first one shows a slight decrease in switching current with increasing magnetic field and a sudden disappearance at high fields, while the second one exhibits a gradual and noticeable reduction of switching currents in magnitude up to $1000 \mathrm{G}$ at which they are totally destroyed. Since Co has some parallel-aligned spins into its magnetic domains even without applied magnetic field, injection of spin polarized current can suppress the superconductivity in some degree resulting in gradual distribution on $I-V$ characteristics. This indicates that oriented spins cause to Cooper pairs to break by killing their coherence between them. Magnetic field enhances the spin directionality inside the Co and the increasing amount of the polarization exposes itself on spectra with stronger diminish the quasiparticle branches in magnitude.
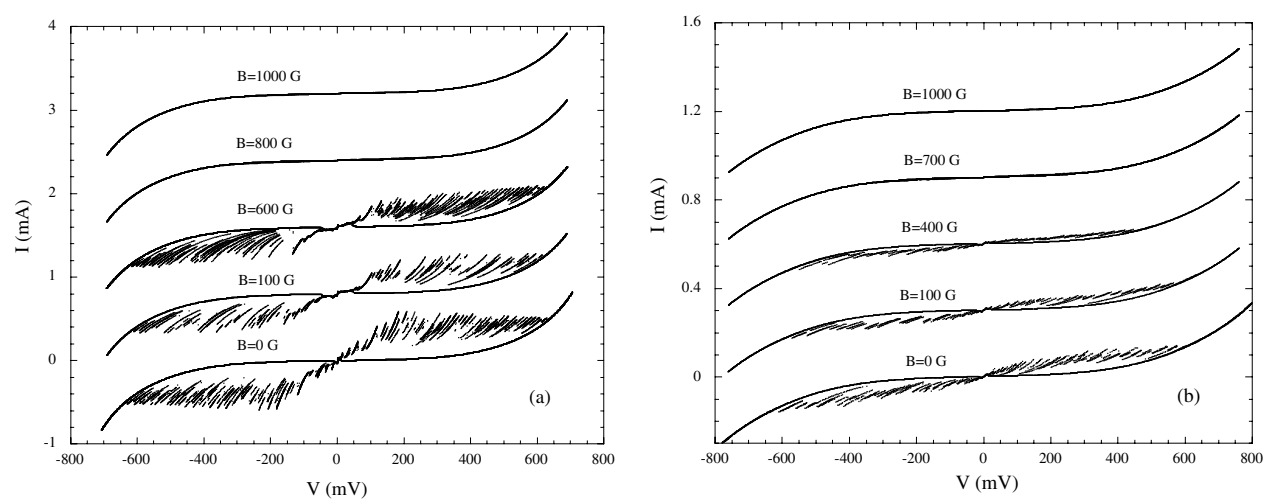

Fig. 2 Magnetic field dependence of $I$ - $V$ characteristics of (a) regular mesa (b) ferromagnetic mesa. The $I$ - $V$ curves are shifted for clarity. 


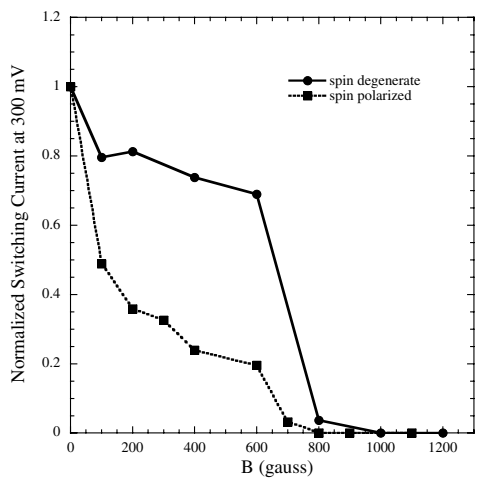

Fig. 3 The comparison of switching currents at $300 \mathrm{mV}$ for regular and ferromagnetic mesas. Note that the return currents are subtracted.

Figure 3 is the comparison of switching currents for two configurations corresponding to $V=300 \mathrm{mV}$ in $I-V$ characteristics as a function of magnetic field. The ferromagnetic mesa data show a much more rapid decrease in switching current with magnetic field around 0-100 G, low field region. The applied field exceeds $\mathrm{H}_{c 1}$ of Bi2212 in this interval for spin degenerate configuration. Apart from this effect, the saturation of magnetization in Co is another reason. Because the magnetization curve of Co showed us, all spins in Co tend to line up with the same direction of external magnetic field above $50 \mathrm{G}$. Between 100 and $600 \mathrm{G}$, switching current slightly changes for spin degenerate configuration, on the other hand there is a discernable decrease in switching currents for spin polarized configurations. There are three effects, spin polarized current, self-field of Co and applied magnetic field, which overlap each other and the resulting tunneling spectra contains collective contribution of these effects.

As a conclusion, IJJ tunneling characteristics show different magnetic field dependences for spin degenerate and polarized currents. At low field, due to the alignment of spins there is a sharp decrease of switching current, which indicates that more polarized current is driven to Bi2212 and the electrons can not find opposite spins to pair up. Making all spins inside cobalt fully-polarized, in other words creating single domain is important, which eliminates the necessity of magnetic field application for spin alignment, hence bare contribution from polarized current can be found.

Acknowledgements The authors acknowledge discussion with K. E. Gray, J. F. Zasadzinski and D. G. Hinks. This work is partially supported by TUBITAK (Scientific and Technical Research Council of Turkey) project number TBAG-2031. L. O. acknowledges support from Turkish Academy of Sciences, in the framework of the Young Scientist Award Program (LO/TUBA-GEBIP/2002-1-17)

\section{References}

[1] R. Kleiner, F. Steinmeyer, G. Kunkel, and P. Muller, Phys. Rev. Lett. 68, 2394 (1992).

[2] L. Ozyuzer, J. F. Zasadzinski, C. Kendziora, and K. E. Gray, Phys. Rev. B 61, 3629 (2000).

[3] L. Ozyuzer, J. F. Zasadzinski, and N. Miyakawa, Int. J. Mod. Phys. B 13, 3721 (1999).

[4] C. Renner, B. Revaz, J.-Y. Genoud, K. Kadowaki, and O. Fischer, Phys. Rev. Lett. 80, 149 (1998).

[5] V. N. Zavaritsky, Phys. Rev. B 72, 094503 (2005).

[6] K. Anagawa, Y. Yamada, T. Shibauchi, and M. Suzuki, Appl. Phys. Lett. 83, 2381 (2003).

[7] L. Ozyuzer, C. Kurter, J. F. Zasadzinski, K. E. Gray, D. G. Hinks, and N. Miyakawa, IEEE Trans. Appl. Supercond. 15, 181 (2005)

[8] X. B. Zhu, Y. F. Wei, S. P. Zhao, G. H. Chen, H. F. Yang, A. Z. Jin, and C. Z. Gu, Phys. Rev. B 73, 224501 (2006). 
[9] A. Yurgens, D. Winkler, T. Claeson, S. J. Hwang, and J. H. Choy, Int. J. Mod. Phys. B 13, 3758 (1999).

[10] J. F. Zasadzinski, L. Ozyuzer, N. Miyakawa, K. E. Gray, D. G. Hinks, and C. Kendziora, Phys. Rev. Lett. 87, 067005 (2001)

[11] D. Bang, H. J. Lee, M. H. Bae, and W. H. Park, Int. J. Mod. Phys. B 19, 199 (2005).

[12] C. Kurter and L. Ozyuzer, J. Opt. Adv. Mater. 7, 415 (2005). 